AIBR

Revista de Antropología

Iberoamericana

www.aibr.org

\section{Volumen 15}

Número 1

Enero - Abril 2020

Pp. $79-104$

Madrid: Antropólogos

Iberoamericanos en Red.

ISSN: 1695-9752

E-ISSN: 1578-9705

\section{La "nueva cultura del aceite» como eje}

\section{de transformación en los territorios olivareros andaluces}

\section{Marta Farré Ribes}

Universidad de Sevilla (US)

\section{Carmen Lozano-Cabedo}

Universidad Nacional de Educación a Distancia (UNED)

\section{Encarnación Aguilar Criado}

Universidad de Sevilla (US)

Recibido: 20.07.2018

Aceptado: 11.12.2018

Dol: 10.11156/aibr.150105

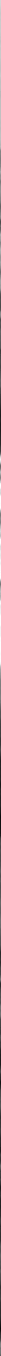




\section{RESUMEN}

En el ámbito del aceite de oliva conviven, actualmente, dos modelos de construcción de la calidad. Un modelo de calidad que se sustenta en los atributos específicos de cada territorio y en el conocimiento tradicional, y otro modelo de calidad basado en normas, estándares y en el conocimiento científico-técnico. La implantación de este segundo modelo implica que las diferentes calidades del aceite de oliva se determinen, por normativa, a través de un análisis sensorial, siguiendo parámetros organolépticos. Este artículo analiza las transformaciones operadas en los territorios olivareros para adaptarse a este modelo de calidad «científico-técnica». Estudia los procesos diferenciales de apropiación de esa «nueva cultura del aceite» en diferentes zonas de Andalucía (España) con una arraigada tradición en la producción de aceite de oliva de calidad. La investigación se ha centrado en tres Sistemas Oleícolas Locales con Denominación de Origen Protegida (Estepa y Puente Genil, Priego de Córdoba y Montoro-Adamuz). En el estudio se ha aplicado una metodología cualitativa, basada en el trabajo de campo realizado durante 12 meses entre 2015-2016. Las técnicas utilizadas han sido la observación participante y las entrevistas semiestructuradas realizadas a 178 actores claves de los tres territorios. Los resultados obtenidos indican que el éxito en el proceso de conversión a esa «nueva cultura del aceite» está vinculado a la territorialización del conocimiento «experto». Resulta fundamental que el panel de cata pase a ser un actor activo del territorio y que se genere un conocimiento accesible que se retroalimente con el conocimiento vernáculo del territorio.

\section{PALABRAS CLAVE}

Calidad alimentaria, aceite de oliva, identidad territorial, Denominación de Origen Protegida, Andalucía.

\section{THE "NEW OLIVE OIL CULTURE" AS A TRANSFORMATION DRIVING FORCE IN ANDALUSIAN OLIVE OIL TERRITORIES}

\section{ABSTRACT}

In the field of olive oil, two quality construction models currently coexist. One quality model based on the specific attributes of each territory and the traditional knowledge and other quality model based on rules and standards and on scientific-technical knowledge. The implementation of this second model implies that the different qualities of olive oil are determined, by regulation, through a sensory analysis, following organoleptic parameters. This article analyzes the transformations carried out in the olive oil territories to adapt to this model of regulated quality. Study the differential processes of appropriation of this "new olive oil culture" in different areas of Andalusia with a tradition rooted in the production of quality oil. The research has focused on three Local Olive Oil Systems of Andalusia that have a Protected Designation of Origin (Estepa y Puente Genil, Priego de Córdoba y Montoro-Adamuz). In the study, qualitative methodology has been applied based on fieldwork carried out during 12 months between 2015-2016. The techniques used were participant observation and semi-structured interviews with 178 key actors from the three territories. Results show that the success in the process of conversion to the "new olive oil culture" is linked to the territorialization of "expert" knowledge. It is essential that the tasting panel becomes an active actor of the territory and that the knowledge generated is accessible and feedback with the vernacular knowledge of the territory.

\section{KEYWORDS}

Food quality, olive oil, territorial identity, Protected Designation of Origin, Andalusia. 


\section{Agradecimientos}

Este artículo forma parte del proyecto: «Las marcas de calidad en el mundo rural: nuevos retos para productores y consumidores» I+D (CSO201342468-P). Grupo de Investigación TECUDE. P.A.I. (SEJ-418).

\section{Introducción}

En los procesos de construcción de la calidad alimentaria conviven dinámicas de territorialización/desterritorialización y estandarización/singularización, que manifiestan la diversidad de respuestas territoriales a la uniformidad de las exigencias globales. En el ámbito del aceite de oliva coexisten dos modelos de calidad: uno que se sustenta en los atributos específicos de cada territorio y en el conocimiento tradicional, y otro basado en normas, estándares y en el conocimiento científico-técnico (Aguilar, 2014; Belletti, Marescotti y Touzard, 2017; Lozano Cabedo, 2011; Quiñones, Penker, Belletti, Marescotti, Scaramuzzi, Barzini, Pircher, Leitgeb y Samper-Gartner, 2016).

El olivar es el agrosistema más representativo y simbólico de Andalucía y constituye un elemento central en la configuración económica, social, cultural e identitaria de esta región española (Consejería de Agricultura, Pesca y Desarrollo Rural, 2015). España ocupa la primera posición mundial en superficie de olivar dedicado a producción de aceite de oliva y Andalucía representa un 60\% de esta superficie en España y un $14 \%$ de la mundial (Observatorio de Precios y Mercados, 2015). La mayor parte de la superficie de olivar en Andalucía está ocupada por el denominado "olivar tradicional». Se conforma de pequeñas parcelas de olivares centenarios ubicadas en zonas de sierra, lo que dificulta la mecanización y la intensificación de su producción. Junto a este se ha desarrollado, en las últimas décadas, el "olivar intensivo o súper intensivo", minoritario pero en crecimiento. Se trata de una producción mecanizada, de gestión empresarial y muy adaptada a las exigencias del mercado (Colombo, Villanueva Perujo y Ruz Carmona, 2016; Sánchez, Garrido y Paniza, 2017).

En la actualidad, conviven dos grandes modelos en los territorios oleícolas: el modelo de cantidad y el modelo de calidad. El primer modelo, que se basa en la producción de aceite de oliva indiferenciado que se comercializa a granel, es el mayoritario y sobre el que se sustenta la economía de este sector. El segundo modelo, centrado en la producción de aceite de oliva envasado de calidad, es minoritario y tiene una escasa repercusión económica, ya que únicamente se envasa, en su fase local, entre 
un 10-20\% del producto obtenido (Alimarket, 2017; Barea y Ruiz Avilés, 2009; Rodríguez Cohard y Parras-Rosa, 2012). Sin embargo, esta pequeña proporción de aceite envasado de calidad ha adquirido relevancia económica y simbólica, a medida que su aportación anual crecía y proliferaban las iniciativas que promueven la multifuncionalidad del olivar y su componente patrimonial: oleoturismo, catas de aceite o museos (Sánchez Martínez y Ortega Ruiz, 2016), así como Denominaciones de Origen Protegidas (DOP) del aceite de oliva. España cuenta actualmente con 29 certificaciones de este tipo, de las cuales 12 se ubican en Andalucía.

El modelo de calidad también ha ido ganando repercusión, a medida que instituciones como la Unión Europea y el Consejo Oleícola Internacional establecían normativas ${ }^{1}$ para estandarizar la calidad del aceite de oliva a través de la creación de diferentes categorías: Aceite de Oliva Virgen Extra, Aceite de Oliva Virgen, y Aceite de Oliva. Es decir, se ha construido una calidad "científico-técnica» sustentada en la presencia de unos parámetros específicos que se detectan mediante análisis fisicoquímicos y organolépticos. El aceite de oliva es actualmente el único producto agroalimentario sujeto, por normativa europea, a un método de análisis sensorial para determinar su categorización según parámetros organolépticos, lo que ha requerido de la creación de paneles de cata, con catadores entrenados según un método regulado, dado que estas entidades son las únicas legitimadas para realizar la clasificación de los aceites.

Por tanto, junto a una calidad «territorial» que responde a los atributos específicos (culturales, sociales, climatológicos, edafológicos, etc.) de cada zona, se ha desarrollado una calidad "científico-técnica» y una «nueva cultura del aceite» (recurriendo a la terminología emic que se utiliza en los territorios oleícolas). La implantación de este segundo modelo ha requerido de la transformación de las prácticas productivas, industriales, comerciales y de consumo de los territorios olivareros. Las prácticas agronómicas tradicionales (manejo no mecanizado, recolección tardía, mezcla de aceitunas de suelo y vuelo ${ }^{2}$, traslado no diario a la almazara ${ }^{3}$ ), las industriales (escasa modernización, baja capacidad de molturación, atroje o almacenamiento de las aceitunas antes de la moltura-

1. El Reglamento (CE) 2568/1991 describe las características químicas y/o sensoriales de los aceites de oliva y de orujo de oliva y sus métodos de análisis. Existen modificaciones en el Reglamento (CE) 1989/2003, Reglamento (CE) 640/2008 respecto al análisis sensorial, Reglamento (CE) 338/2013 referente a los métodos de análisis. Respecto al COI, el Reglamento T.15/nº $3 /$ Rev. 8 establece la clasificación comercial (COI, 2105a) y el Reglamento T.20/n $15 /$ Rev.7 se refiere al análisis sensorial.

2. La aceituna de «vuelo» es la que se derriba del árbol y se entrega en la almazara sin haber tocado el suelo.

3. La almazara es el molino o industria donde se obtiene el aceite moliendo la aceituna. 
ción) y las comerciales (comercialización de granel indiferenciado), entran en conflicto con las exigencias de la conversión a la producción de calidad «reglamentada». Estos criterios exigen aceitunas que no estén dañadas y que en su recolección, traslado y transformación se conserve al máximo la pureza fisicoquímica y organoléptica del aceite. Ello ha implicado, en el ámbito productivo, la mecanización del proceso, la recolección temprana separando la aceituna de vuelo de la de suelo y el traslado diario del producto a la almazara. En el ámbito industrial, ha conllevado la modernización de las almazaras, el aumento de su capacidad y la molturación diaria, mientras que en el comercial ha supuesto la ampliación del porcentaje de aceite virgen extra envasado. La extensión de esta "nueva cultura del aceite» también ha incidido en la reconfiguración de las relaciones de poder en el sector oleícola, en la transformación de los procesos de identificación local y en las dinámicas de gobernanza de los territorios olivareros.

La literatura académica ha prestado especial atención a la calidad «territorial» a través del análisis de los procesos de singularización de la producción a través de Denominaciones de Origen Protegidas (DOP) e Indicaciones Geográficas Protegidas (IGP) (Belletti, Marescotti y Brazzini, 2014; Belletti, Marescotti y Touzard, 2017; Lozano Cabedo, 2013; Quiñones et al., 2016; Fernández-Zarza, Amaya-Corchuelo y AguilarCriado, en prensa). Esta línea de análisis ha destacado la aportación de la calidad diferenciada al desarrollo rural en Andalucía (Cendón, 2016; Sánchez-Escobar, 2015; Sanz-Cañada, Hervás, Sánchez-Escobar y CoqHuelva, 2014). Especial atención se ha otorgado al análisis de las relaciones de poder que subyacen a la implantación de estas marcas de calidad (López-Moreno, Aguilar-Criado, Lozano-Cabedo y Pérez-Chueca, 2015; López-Moreno y Aguilar-Criado, 2015), y a la transferencia de innovación y conocimiento que se está produciendo en los Sistemas Oleícolas Locales, a través de la teoría de las convenciones y el análisis de redes (Cendón, Sanz-Cañada y Lucena-Piquero, 2014; Coq-Huelva, Sanz-Cañada y Sánchez Escobar, 2014; Sanz-Cañada y Macías Vázquez, 2005).

Sin embargo, apenas se ha atendido a las profundas transformaciones culturales que ha implicado la implantación de esa «nueva cultura del aceite». Este artículo analiza los procesos diferenciales de apropiación del conocimiento científico-técnico que se dan en los territorios olivareros. A partir de la identificación de tres modelos, exploramos cómo se combinan, en cada uno de los territorios, el conocimiento vernáculo y el «experto", así como la tradición, encarnada en la industria almazarera local, y la innovación, asociada al mercado y a la cultura empresarial. En segundo lugar, analizamos las diferentes políticas de calidad instauradas en los 
territorios, las entidades que las lideran y el papel de las marcas territoriales y comerciales en dichas políticas. En tercer lugar, estudiamos aquellos elementos en torno a los cuales se construye, en cada territorio, el relato de la puesta en valor del aceite de oliva.

\section{Método}

Para esta investigación, elegimos tres unidades de observación en Andalucía que responden a Sistemas Oleícolas Locales con larga trayectoria en la producción de aceite de oliva de calidad: la comarca de Estepa y Puente Genil (ubicada entre las provincias de Sevilla y Córdoba), la comarca de Priego de Córdoba y la comarca de Montoro-Adamuz (ambas en la provincia de Córdoba). Estas tres zonas fueron elegidas por poseer una asentada y dilatada historia en la producción olivarera, pero, especialmente, por haber seguido itinerarios distintos en sus respectivos procesos de conversión a la producción de calidad (Mapa 1).

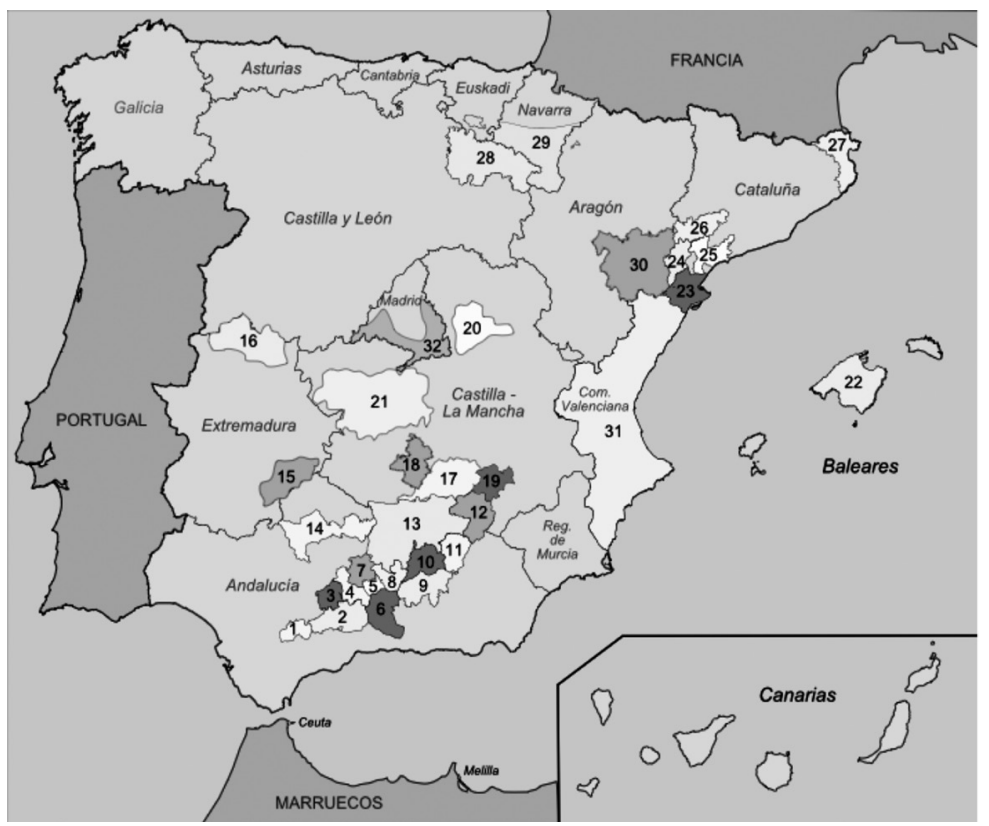

Mapa 1: Mapa de las DOP de aceite en España. № 3: DOP Estepa y Puente Genil. № 5 : DOP Priego de Córdoba. No 14: DOP Montoro-Adamuz (Fuente: https://upload.wikimedia. org/wikipedia/commons/d/d8/Olive_oil_Spain.svg. Accedido el 4 de julio de 2018). 
El estudio responde a una metodología cualitativa, basado en el trabajo de campo intensivo realizado durante 12 meses entre los años 20152016. Las técnicas utilizadas han sido la observación participante y las entrevistas semiestructuradas a actores claves del territorio: olivareros, técnicos de empresas (privadas y cooperativas), gerentes de empresas (privadas y cooperativas) y representantes institucionales (Tabla 1).

\begin{tabular}{|l|r|r|r|r|r|}
\hline & Olivareros & $\begin{array}{c}\text { Técnicos } \\
\text { institucio- } \\
\text { nales y } \\
\text { empresas }\end{array}$ & $\begin{array}{c}\text { Gerentes } \\
\text { y presidentes } \\
\text { de empresas }\end{array}$ & $\begin{array}{c}\text { Representan- } \\
\text { tes Institu- } \\
\text { cionales }\end{array}$ & Total \\
\hline $\begin{array}{l}\text { Estepa y } \\
\text { Puente Genil }\end{array}$ & 35 & 40 & 12 & 11 & 98 \\
\hline $\begin{array}{l}\text { Priego de } \\
\text { Córdoba }\end{array}$ & 13 & 22 & 14 & 5 & 54 \\
\hline $\begin{array}{l}\text { Montoro- } \\
\text { Adamuz }\end{array}$ & 7 & 8 & 7 & 4 & 26 \\
\hline Total & 55 & 70 & 33 & $\mathbf{2 0}$ & 178 \\
\hline
\end{tabular}

Tabla 1: Número de entrevistas realizadas a los diferentes actores clave del territorio (elaboración propia).

Las entrevistas se estructuraron en torno a tres bloques de preguntas. El primer bloque indagaba en qué consiste, en opinión de los actores del territorio, la calidad del aceite de oliva. El segundo bloque se centró en conocer los principales hitos que definen el proceso territorial de transformación a la calidad. El tercer bloque exploró las dimensiones y los actores que han favorecido y dificultado dicho proceso.

\section{Análisis y discusión de resultados}

El análisis de la trayectoria y las dinámicas seguidas en cada uno de los territorios seleccionados nos ha permitido identificar tres modelos específicos de construcción de la calidad. En el Cuadro 1, vemos que cada uno de ellos presenta características diferenciales en las dimensiones estudiadas. A lo largo de este epígrafe, describiremos brevemente cada uno de estos modelos centrándonos en las cuestiones que están directamente vinculadas con los objetivos de este texto. 


\begin{tabular}{|c|c|c|c|}
\hline & \multicolumn{3}{|c|}{ MODELOS DE CONSTRUCCIÓN DE LA CALIDAD } \\
\hline & Calidad Corporativa & Calidad Territorial & $\begin{array}{c}\text { Calidad como } \\
\text { Expectativa Futuro }\end{array}$ \\
\hline $\begin{array}{l}\text { DOP con la que se } \\
\text { corresponde }\end{array}$ & $\begin{array}{l}\text { Estepa y Puente } \\
\text { Genil }\end{array}$ & Priego de Córdoba & Montoro-Adamuz \\
\hline Entidad líder & $\begin{array}{l}\text { Cooperativa Segundo } \\
\text { Grado Oleoestepa }\end{array}$ & $\begin{array}{l}\text { Consejo Regulador } \\
\text { DOP y sector tu- } \\
\text { rístico }\end{array}$ & $\begin{array}{l}\text { Agentes políticos } \\
\text { y Grupo } \\
\text { de Desarrollo Rural }\end{array}$ \\
\hline $\begin{array}{l}\text { Entidad } \\
\text { que controla } \\
\text { el Panel Cata }\end{array}$ & $\begin{array}{l}\text { Cooperativa Segundo } \\
\text { Grado Oleoestepa }\end{array}$ & $\begin{array}{l}\text { Consejo Regulador } \\
\text { DOP }\end{array}$ & $\begin{array}{l}\text { Cooperativa } \\
\text { Segundo Grado } \\
\text { DCOOP }\end{array}$ \\
\hline $\begin{array}{l}\text { Papel de la DOP } \\
\text { en modelo de } \\
\text { calidad }\end{array}$ & Secundario & Preponderante & Incipiente \\
\hline $\begin{array}{l}\text { Eje de su modelo } \\
\text { de calidad }\end{array}$ & $\begin{array}{l}\text { Adecuación al mode- } \\
\text { lo «científico-técni- } \\
\text { co» de calidad }\end{array}$ & $\begin{array}{l}\text { Características } \\
\text { biofísicas del terri- } \\
\text { torio, conocimiento } \\
\text { vernáculo y experto }\end{array}$ & $\begin{array}{l}\text { Variedad local } \\
\text { de aceituna } \\
\text { y patrimonio } \\
\text { material }\end{array}$ \\
\hline $\begin{array}{l}\text { Integración } \\
\text { conocimiento } \\
\text { experto }\end{array}$ & $\begin{array}{l}\text { «Expertización» } \\
\text { sector }\end{array}$ & $\begin{array}{l}\text { «Expertización» } \\
\text { territorio }\end{array}$ & $\begin{array}{l}\text { Islas } \\
\text { de conocimiento }\end{array}$ \\
\hline $\begin{array}{l}\text { Estrategia } \\
\text { de Marca }\end{array}$ & $\begin{array}{l}\text { Una marca comercial } \\
\text { ejerce como marca } \\
\text { territorial }\end{array}$ & $\begin{array}{l}\text { Una marca territo- } \\
\text { rial aglutina plu- } \\
\text { ralidad de marcas } \\
\text { comerciales }\end{array}$ & $\begin{array}{l}\text { Ausencia de marca } \\
\text { territorial } \\
\text { representativa }\end{array}$ \\
\hline $\begin{array}{l}\text { Función } \\
\text { de la marca } \\
\text { a nivel identitario }\end{array}$ & Identidad corporativa & Identidad territorial & Identidad difusa \\
\hline $\begin{array}{l}\text { Gobernanza } \\
\text { territorial }\end{array}$ & Vertical y privada & $\begin{array}{l}\text { Vertical y horizon- } \\
\text { tal, público-privada }\end{array}$ & $\begin{array}{l}\text { Fragmentada, } \\
\text { público-privada }\end{array}$ \\
\hline
\end{tabular}

Cuadro 1. Aspectos clave en la construcción de la calidad en los diferentes modelos (elaboración propia).

\subsection{Tres modelos territoriales de construcción de la calidad. Características principales}

El primer modelo es el denominado "Calidad Corporativa», representado por la comarca de Estepa y Puente Genil. En este territorio, el 
proceso de conversión a la producción de calidad «científico-técnica» ha estado liderado por una Cooperativa de Segundo Grado: Oleoestepa. Esta entidad, dedicada al envasado y la comercialización del aceite producido en el territorio, es la que ha liderado, desde su creación en 1987, este proyecto comarcal, con un claro protagonismo del sector cooperativo, mayoritario en la comarca. El envasado y la comercialización de la mayor parte del aceite producido en el territorio se realizan a través Oleoestepa, a excepción de una almazara cooperativa y dos almazaras privadas que envasan y comercializan su propio aceite.

Desde esta entidad se impulsó, en 2005, la creación de la DOP Estepa y Puente Genil. De las 21 entidades asociadas a la DOP (que suponen el $95 \%$ de las entidades del territorio), 18 son cooperativas pertenecientes a Oleoestepa. Las tres entidades restantes son la cooperativa y las dos almazaras privadas que, como hemos mencionado, no pertenecen a Oleoestepa. Su protagonismo también es patente en el ámbito de las marcas, pues de las cuatro marcas que se comercializan con DOP, tres pertenecen a Oleoestepa y únicamente una pertenece a una cooperativa que está fuera de la Cooperativa de Segundo Grado.

Esta entidad ha impulsado una potente cultura corporativa y un modelo de gobernanza vertical y privado, centrado exclusivamente en el sector oleícola. Oleoestepa ha marcado también las directrices del modelo de calidad a seguir en la comarca, mientras que la DOP quedaba relegada a un segundo plano. Y ello se percibe claramente por el hecho que la marca comercial de la Cooperativa de Segundo Grado (denominada también «Oleoestepa») se instaura como hegemónica en el territorio. Ello le ha permitido, a nivel externo, promocionar el territorio a través de su marca corporativa que ejerce, a su vez, como marca territorial, fomentando el sentimiento de pertenencia colectiva del sector al proyecto de calidad. Y, a nivel interno, liderar la difusión de la «cultura del aceite» y generar una identidad territorial alrededor de su modelo de calidad. 


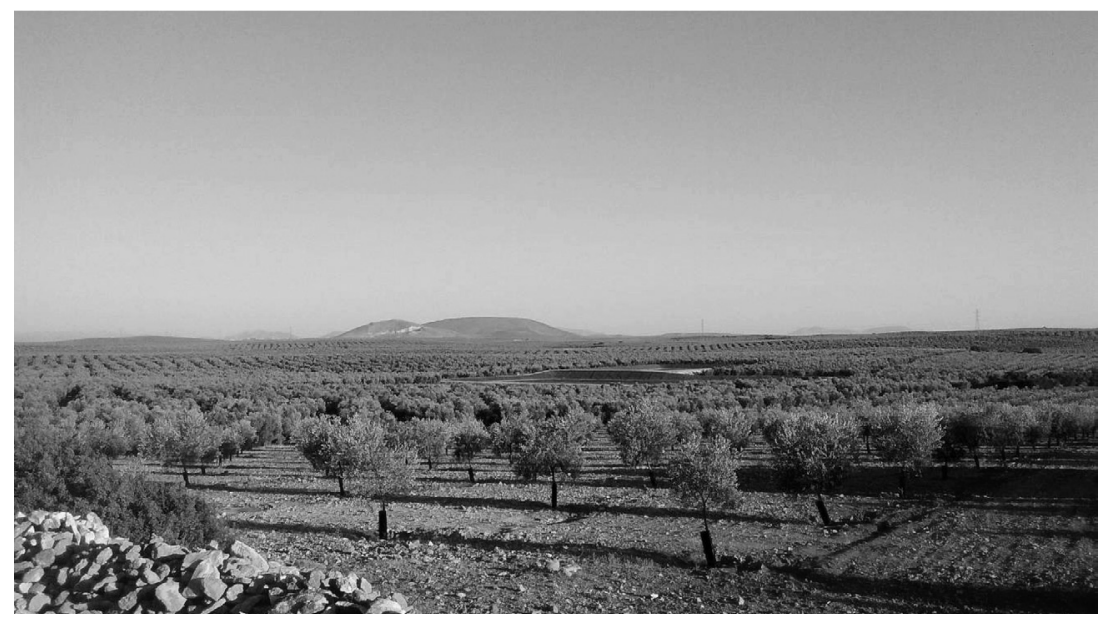

Foto 1. Olivares de nueva plantación. DOP Estepa y Puente Genil (elaboración propia).

El segundo modelo es el denominado «Calidad Territorial», representado por la comarca de Priego de Córdoba. En este modelo coexisten múltiples entidades: almazaras privadas, cooperativas, envasadoras y comercializadoras, así como diversidad de políticas de calidad, de estrategias de comercialización y de marcas comerciales. La entidad que lideró el proceso de conversión a la producción de calidad fue la DOP Priego de Córdoba, ya que es la que representa y en la que convergen la diversidad de iniciativas del territorio. La DOP fue creada el año 1995 a iniciativa del propio sector, con liderazgo tanto del sector cooperativo como del privado. Entre las 24 entidades vinculadas al sector del aceite que forman parte de la DOP hay una asociación de cooperativas de primer grado unidas para la comercialización, cuatro cooperativas (de las cuales una está inscrita también como envasadora y comercializadora), seis almazaras privadas (dos de las cuales están inscritas también como envasadoras y comercializadoras), así como cuatro envasadoras y comercializadoras. Esta entidad aglutina a la mayor parte del sector (79\% de los operadores), ya que únicamente cinco entidades no forman parte de la DOP (dos almazaras cooperativas y tres iniciativas privadas).

En la DOP hay inscritas 11 marcas repartidas entre los diferentes operadores, pues solo dos de ellas pertenecen a una misma entidad. Estas marcas están amparadas bajo una única marca territorial representada por la DOP. El modelo de gobernanza posee un carácter público-privado, ya que la colaboración entre ambos sectores es estrecha y fluida. Pero también una dimensión vertical y horizontal, dado que no solo se ha 
centrado en el sector oleícola, sino que ha impulsado el trabajo en red con otros sectores económicos y con otras instituciones y actores del territorio. La DOP es también la que lidera la difusión de la «cultura del aceite» a nivel interno y externo, como veremos con mayor detalle, promoviendo una identidad colectiva del territorio en la que la «expertización» en el aceite de calidad pasa a ser el eje central.

El tercer modelo, denominado "Calidad como Expectativa de Futuro», está representado por el territorio integrado en la DOP MontoroAdamuz, creada en 2007 por algunos políticos del territorio y por el Grupo de Desarrollo Rural. La DOP integra solo a la mitad de las entidades del sector oleícola comarcal (11 de 20), aunque está representado todo el sector cooperativo de la zona, compuesto por cinco almazaras y por la Cooperativa de Segundo Grado. El resto de entidades inscritas en la DOP son dos almazaras privadas y tres envasadores.

La política de calidad del territorio está dirigida por DCOOP, que es la Cooperativa de Segundo Grado que comercializa todo el aceite producido por las cooperativas de la comarca. Esta entidad se caracteriza por tener presencia en múltiples territorios olivareros (es el mayor productor mundial de aceite de oliva y de aceitunas de mesa, con 400.000 hectáreas de olivar y 50 millones de olivos) y en múltiples sectores (sector del vino, ganadería, cereales o los suministros, y la forman 65.000 agricultores y ganaderos del sur de España). Por tanto, el proceso de producción de aceite en la zona está liderado por una entidad desterritorializada, con una política enfocada al modelo de la cantidad, el crecimiento en volumen y la incorporación de nuevas cooperativas y productos. Sin embargo, una parte de su estrategia se orienta a la producción de aceite de calidad, dado que hay un segmento de mercado que lo demanda. De ahí que la entidad colabore con la DOP Montoro-Adamuz ya que, a través de las cinco marcas que DCOOP tiene inscritas en la DOP, se comercializa prácticamente la totalidad de aceite calificado del territorio. Una colaboración asimétrica, pues la DOP tiene, actualmente, un papel minoritario en la difusión de la «cultura del aceite» y cuenta con poco apoyo territorial. No existe, por tanto, una gobernanza territorial que cohesione ni al sector oleícola ni al territorio, y ello se percibe en el hecho que no existe una marca, ni de carácter territorial ni comercial, que represente a la zona.

\subsection{La calidad estandarizada y la apropiación local del conocimiento "experto»}

Los discursos y las prácticas que se construyen alrededor de la calidad «científico-técnica» responden a un proceso de objetivación y, supuesta- 
mente, están desvinculados de las especificidades territoriales y del arraigo histórico de la producción olivarera. Sin embargo, como veremos en este apartado, este conocimiento estandarizado es percibido, practicado, institucionalizado, integrado en la memoria colectiva o difundido a nivel local de manera diferencial en los diferentes territorios.

El modelo "Calidad Corporativa» se caracteriza por haber hecho suyos los criterios de calidad "científico-técnicos» y por desarrollar una política de «expertización» del sector oleícola en la producción de calidad estandarizada. De hecho, la génesis de Oleoestepa está directamente ligada a la inquietud de determinadas personas vinculadas al sector olivarero de la comarca, que pensaban que el territorio debía generar su propio conocimiento y regular la producción de aceite de calidad. El objetivo que se marcaron fue conseguir que todo el aceite producido en el territorio cumpliera con los estándares requeridos para poder ser clasificado en la categoría de mayor calidad: Aceite de Oliva Virgen Extra. Ello pasaba por unificar el nivel de calidad, lo que requirió de la adaptación de las prácticas, manejos y saberes desarrollados tradicionalmente en el territorio, pero también de la construcción y difusión de una nueva «cultura del aceite», a través de la profesionalización del sector comarcal en los nuevos requerimientos de producción de calidad, y de la ubicación del laboratorio y el panel de cata en el territorio y, más concretamente, en las instalaciones de Oleoestepa.

En este proceso, el panel de cata se ha convertido en un actor clave en las relaciones de poder, pues, como señalan los informantes, es el «corazón» y la "piedra angular» de Oleoestepa: "Al tener el panel de cata [...] se sabe catalogar, entonces, a los diferentes certámenes, está la jefa del panel de cata. Y es la jefa y lleva abi muchos años. Y esta señora es la que dice [...] y es la que decide toda la calidad» (agricultor, comarca de Estepa y Puente Genil). Ello ha permitido a esta entidad controlar el proceso de tipificación de aceite y detentar el poder de marcar los precios, tanto a nivel interno con las cooperativas, como externo con los distribuidores y el mercado.

La apropiación del conocimiento «experto» por parte todos los eslabones del sector oleícola del territorio ha conllevado también reformulaciones identitarias. A nivel individual, la identidad de los agricultores ya no se sustenta solo en los tradicionales vínculos familiares y comunitarios, sino también en la satisfacción de haber sido capaces de adaptarse a las directrices de profesionalización y modernización de las prácticas productivas e industriales. A nivel colectivo, se manifiesta en el sentimiento de resistencia contra el lobby de la gran industria del aceite por poner en entredicho la rigurosidad científico-técnica de las certificaciones emitidas por el panel de cata. 
El modelo "Calidad Territorial» se caracteriza por una política de «expertización» del territorio destinada especialmente a formar a la población (no solo a los miembros del sector oleícola) y al sector turístico. Esta estrategia de territorialización del conocimiento «experto» ha estado liderada por la DOP Priego de Córdoba, que es la que ostenta el panel de cata. De ahí la disociación entre el poder de categorizar los aceites (en manos de la DOP) y la de poner el precio al producto (que gestionan las comercializadoras del territorio).

La apropiación local de ese conocimiento «experto" se ha realizado fundamentalmente a través de la formación en la cata de aceites. A través de cursos, premios y/o acreditaciones se han generado nuevas figuras/ agentes profesionales, que pasan a ser importantes referentes locales: los expertos catadores. De hecho, existe por parte de la DOP una política estratégica para formar desde jóvenes a la población para que sepa poner en valor su producto. La «Escuela de Cata» de la entidad es un claro ejemplo de esta voluntad formativa.

\footnotetext{
Nuestro objetivo es cuanta más gente formada en torno la calidad, pues al final sabremos diferenciar y que luego cada consumidor, pues decida. Hay que estar formado en este mundo, no nos quedamos con el conocimiento dentro [...] Y esa función la hacemos, buscamos hueco de 15 o 20 minutos, para que las personas se vayan con eso, conocimiento, que al final son los que nos dan a conocer (técnico, Priego de Córdoba).
}

El panel de cata desempeña diversas funciones: es clave en la «expertización» del territorio, es un instrumento de difusión del conocimiento del aceite al visitante y es un vehículo de profundos intercambios culturales a través del encuentro/comunicación/relación entre locales y visitantes, conocimiento «local»y «experto», formado y formador, sectores económicos diversos e instituciones. Pero, además, está reconfigurando las relaciones de poder en la cadena agroalimentaria. Ello se hace evidente cuando la gran industria del aceite demanda los servicios de profesionales catadores del territorio para que formen a sus equipos de calidad y/o trabajen como técnicos de cata en la selección de aceites. Y cuando estos profesionales son requeridos como formadores por Centros de Investigación, Agrupaciones Agrarias, DOP, o como miembros de jurados para concursos de cata y premios de aceite.

Esta estrategia está fomentando que, a nivel identitario, todo el territorio muestre el orgullo de reconocerse en la capacidad de transmitir un «nuevo» conocimiento «experto» y la satisfacción por poder «enseñar», «mostrar», "introducir» al "otro», en el «mundo del aceite». "Esto es una pasión lo que se siente, ganas que la gente conozca como tú conoces el 
tema, se trata de transmitir ese sentimiento, ese conocimiento. Es que la gente no sabe, que la gente aprenda» (experto catador y agricultor, Priego de Córdoba).

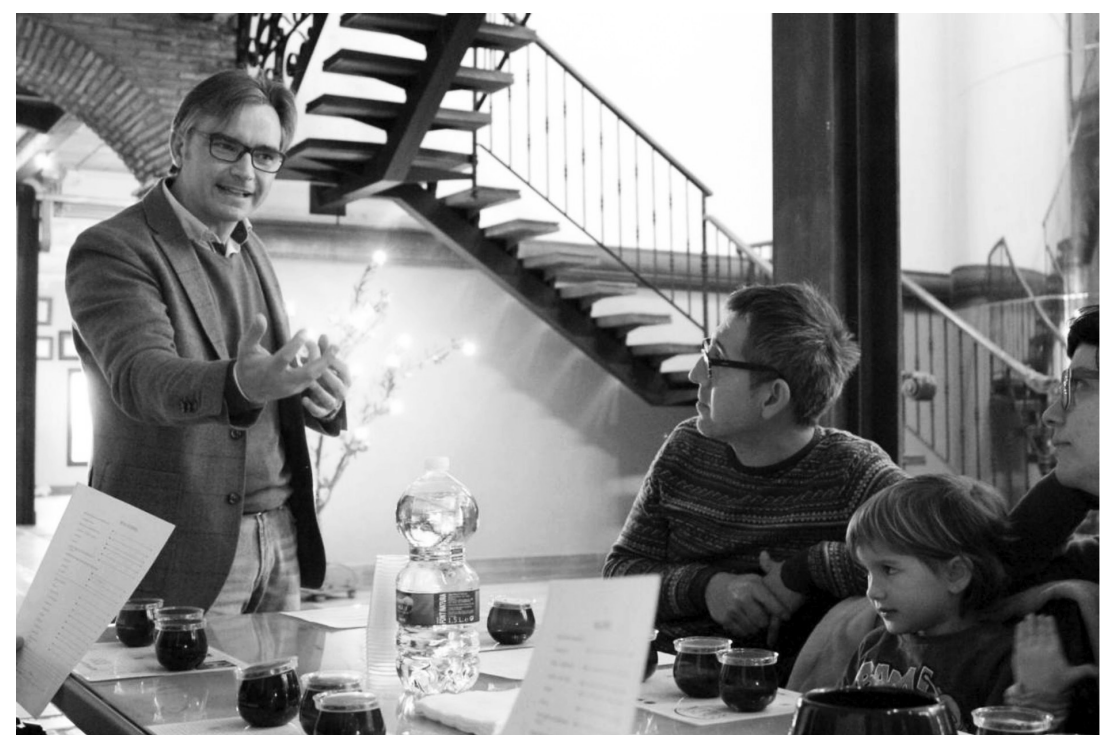

Foto 2. Cata de aceites para turistas. DOP Priego de Córdoba (elaboración propia).

El modelo «Calidad como Expectativa de Futuro» se caracteriza por la dependencia y la desconexión territorial respecto a los procesos científico-técnicos determinantes en la producción de calidad "reglamentada». En este caso, la categorización de la calidad de los aceites de la zona depende de un panel de cata que es ajeno al territorio y que está inserto en una de las grandes corporaciones olivareras: la Cooperativa de Segundo Grado DCOOP. La distancia, física y simbólica, con el panel de cata propicia que no se generen todas las sinergias territoriales que hemos visto en los dos casos anteriores. La ausencia de políticas de difusión de la «nueva cultura del aceite» a nivel local, también influye en el desconocimiento del sector cooperativo respecto al saber «científico-técnico».

Existen determinadas iniciativas que tratan de incorporar el saber «experto» al territorio, aunque tienen un carácter fragmentado y aislado. Las experiencias que han apostado por la producción de Aceite de Oliva Virgen Extra de alta calidad se caracterizan por ser iniciativas privadas innovadoras lideradas por personas que provienen de otros sectores económicos y/o de otros territorios. Resulta llamativo que la distancia con 
respecto a la tradición olivarera de la zona sea un elemento facilitador para introducir los nuevos valores asociados a la calidad, poner en valor el territorio y transformar las prácticas productivas. Otra de las entidades que ha promovido la integración del conocimiento «experto» en el territorio ha sido Patrimonio Comunal Olivarero, que posee en Montoro un laboratorio y un panel de cata que da servicio a las iniciativas privadas innovadoras presentes en territorio y que desarrolla algunos cursos de análisis sensorial del aceite. Sin embargo, sus acciones no están incidiendo en la apropiación local del saber "experto", tal y como sí ocurre en los dos casos anteriores. Asimismo, ni la DOP ni la Cooperativa de Segundo Grado tienen una política prioritaria de territorialización del conocimiento «experto». Una situación que coloca al territorio en una posición de subordinación con respecto a los procesos globales de producción y difusión de conocimiento en torno a la calidad «estandarizada».

\subsection{El papel de la tradición y la innovación en el proceso territorial de construcción de la calidad del aceite de oliva}

Los tres modelos despliegan diferentes políticas de construcción de la calidad y aplican diversos mecanismos para generar cohesión en torno al proyecto de calidad hegemónico: pluralidad de políticas marquistas, diferente presencia de marcas comerciales y territoriales, desigual implicación de las DOP y de otros sectores económicos y actores del territorio, así como mayor o menor conciencia de transformación productiva comarcal. Pero todas ellas se sustentan en la articulación específica entre conocimiento vernáculo y experto, y en la combinación de elementos tradicionales e innovadores.

En el modelo "Calidad Corporativa» destaca la conciencia que tiene el sector oleícola de haber vivido un proceso de transformación cultural radical desde el modelo «tradicional» hacia el modelo "científico-técnico». En los discursos se enfatiza el cambio de cosmovisión, de valores y de mentalidad que ha implicado el proceso de conversión hacia la calidad a través de la utilización de términos como: «educar», «enseñar», "convencer», «adiestrar», «absorber», «aprender», «confiar», «asimilar», «transmitir», «concienciar», «inferir», «transformar», «cambiar», «revolución cultural», «conversión», «mentalidad» o «ideología».

El aceite [virgen extra] es un producto nuevo. Tiene 30 años y los estamos educando. Aquí los estamos educando a marchas forzadas, más rápidamente y con mayor inversión y en otros sitios más lentamente, pero aquí vamos a marchas forzadas: virgen extra, virgen extra, virgen extra. No hablamos de 
aceite de oliva, [sino de] virgen extra. Con muchos requisitos hacia los agricultores, para que lo que produzcan sea virgen extra (técnico, Estepa y Puente Genil).

Existe un único mito de origen de la transformación a la producción de calidad "científico-técnica», con unos personajes claros, referentes del cambio. Estas personas, que impulsaron la creación de Oleoestepa, fomentaron la territorialización del panel de cata y han difundido la «nueva cultura del aceite», se erigen como símbolos de la entidad y del territorio. Y ello se percibe en el papel preponderante que desempeñan en los rituales que refuerzan la pertenencia al proyecto (asambleas, entregas de premios, conferencias, ferias, etc.), o en el hecho de que las nuevas instituciones que se crean en la comarca reciban su nombre (Cátedra José Humanes).

Como hemos visto, el eje fundamental en el relato de la calidad de este modelo ha sido la adecuación a las normas y estándares, así como la innovación en prácticas y saberes. Un relato que se ha concretado en unas normas de régimen interno de obligado cumplimiento por todas las cooperativas, así como en una política de pago por calidad. En un contexto de inestabilidad de precios, Oleoestepa estableció una política estable de precios según calidades, que incentivó a agricultores y almazaras a cambiar sus prácticas y a ajustarse a los requisitos de la producción estandarizada. Ahora bien, la incorporación de estas normas ha sido el resultado de un proceso de tensión/diálogo, sobre todo con la red de cooperativas de primer grado, que son las que encarnan los valores tradicionales y constituyen el principal referente identitario local. Actores con fuerte arraigo y autoridad dentro de las cooperativas, como presidentes, gerentes y, especialmente, maestros de almazara son las figuras que encarnan los valores tradicionales. Y esa reputación es la que ha permitido que se conviertan en los principales nodos de difusión de la política de calidad «estandarizada».

Esta dualidad entre lo moderno y lo tradicional ha sido el motor de cambio en la comarca, ya que ha generado una red bidireccional de flujo de conocimiento, aunque también ha propiciado la construcción de un discurso ambivalente. Se considera que la tradición ha sido la base del éxito de la instauración del proyecto de calidad comarcal. Pero también simboliza un «antes», en el que las cosas se hacían «mal», que contrasta con un «ahora», en que se hacen «bien». Para muchos de los entrevistados, las prácticas agronómicas, industriales, comerciales y de autoconsumo tradicionales se perciben como un freno para la transformación a la calidad «científico-técnica». 
Yo siempre digo, cuando vienen aquí los consumidores, las visitas, lo que buscan es romanticismo. Esa leyenda romántica de cómo se hace en la tierra, la piedra, si es posible el burro, qué bonita esa esencia [...] Volvemos ligado con lo de mi cooperativa de toda la vida, de mi pueblo, el aceite de toda la vida [...] Falso, no hay nada más malo que el aceite de toda la vida (técnico, Estepa y Puente Genil).

Esta dualidad se aprecia también en el discurso local sobre las marcas. Existe un fuerte arraigo, entre los olivareros, a la marca de cada una de las cooperativas, pues están vinculadas en el imaginario al autoconsumo y a un sentimiento de identidad local. Pero también se identifican con la nueva marca introducida por la Cooperativa de Segundo Grado, al ser la imagen del nuevo proyecto colectivo de producción de calidad en el territorio.

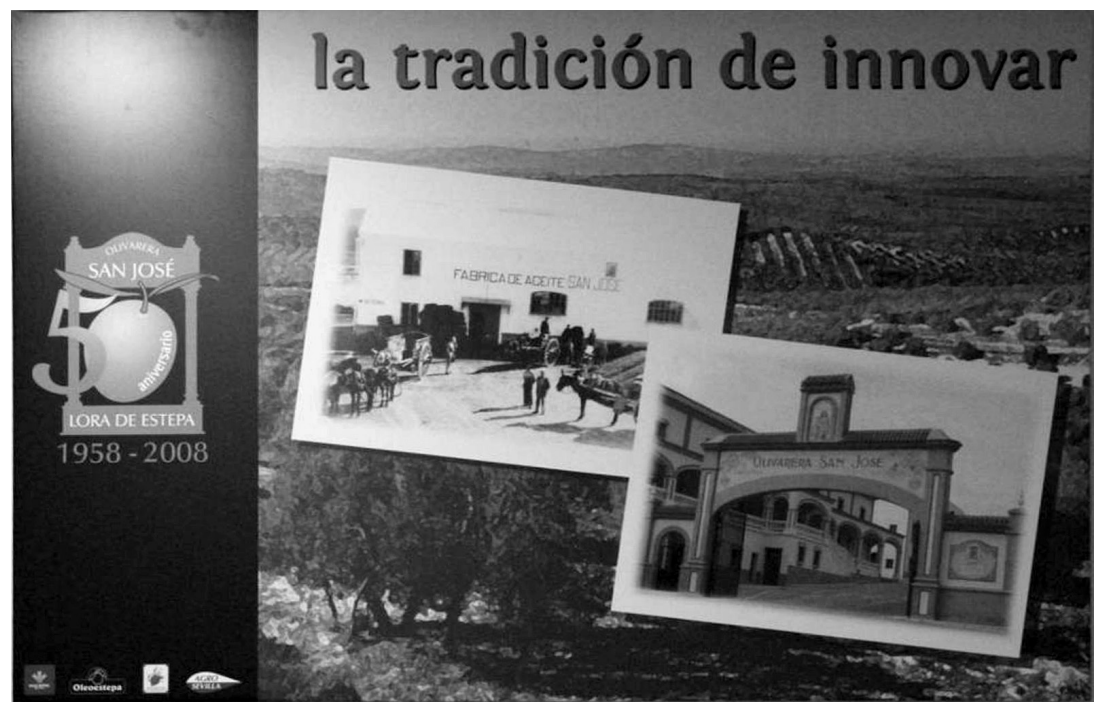

Foto 3. Cartel promocional ubicado en una cooperativa, DOP Estepa y Puente Genil (elaboración propia).

En el modelo «Calidad Territorial» se combinan las referencias a la «expertización» del sector y del territorio con el énfasis en la importancia del saber-hacer tradicional. El proceso de conversión hacia la producción de calidad se sustenta, según los actores locales, en dos elementos. En las características específicas de las aceitunas producidas en la Sierra de la Tiñosa, una zona del territorio que presenta unas determinadas condicio- 
nes biofísicas y microclimáticas que otorgan unas cualidades diferenciales al aceite. Pero también en el conocimiento tradicional del sector industrial $y$, especialmente, en el saber hacer de determinados maestros de almazara que poseen la capacidad de detectar las fincas en las que la materia prima es de mayor calidad. La negociación entre olivareros y almazaras para adquirir esta producción de calidad se produce durante un período concreto en el que se aplica el conocido como «Plan de Calidad», que permite que los pagos se establezcan por calidad y no siguiendo las directrices del mercado.

La apuesta que hace este modelo por una política de calidad plural se ve respaldada por la multitud de premios obtenidos por las marcas de la zona, que aportan valor al global de la producción, así como al territorio en su conjunto. La marca, el posicionamiento, el reconocimiento y la historia de las empresas se definen a través de los premios. Pero estos también tienen una relevancia capital en la construcción de la nueva identidad territorial en torno la DOP porque constituyen un reconocimiento a las prácticas y al saber hacer tradicional, son una importante herramienta de comunicación con el «entorno», fuente de orgullo y reconocimiento por el trabajo «bien hecho».

La política marquista, a diferencia del modelo anterior, se sustenta en una diversidad de marcas. Y si bien es cierto que no existe una entidad líder en este ámbito, son las entidades — públicas o privadas- con más capacidad de inversión en marca y en premios, las que tienen más peso a la hora de establecer cuál es la imagen del territorio y, por tanto, las que tienen más poder en la reconfiguración de las significaciones territoriales. Un modelo de calidad en el que los agricultores no parecen estar del todo integrados, pues consideran que este responde más a los intereses de los industriales que a los del sector olivarero.

Sí, y las seleccionaba [el maestro de almazara] y decía: "esta, esta es la que quiero que me traiga». Y voy a mitad del otoño con la mía, a último de verano, me dice: "se me coge de este trozo". Le digo: "pero si de aquí abajo es más gorda». «iNo!, este». Y yo: "ipero si aquí está más cargado!». Y me dice: «no, coges de este trozo". Le digo: "¿por qué?». Y me dice: "porque la más gorda tiene más agua. Y entonces cuando ya la muelen, la pulpa no es, como te digo yo, que pierde ya un poco de aroma». Pero esto, lo mismo estaba cargado y vio la aceituna como te digo yo, la vio vigorosa. Que no pasó sed en el verano. Y digo: «bueno ya está, de aquí te cojo yo». Y empecé a coger. Y cogió ese año le entró 100 mil kilos. Fue finca por finca y dijo 100 mil kilos. Yo le llevé 25 mil. Le llevé una cuarta parte de lo que seleccionó. Ese año le dieron premio. El León de Oro en Italia. Estaba un día en la oficina, había unos cuantos de la directiva. Y digo yo: «bueno, el León de Oro, el rabo será de mi finca». Y dicen, y dice: «¿el rabo?». Y digo: "hombre, del premio tengo yo que extraer una 
cuarta parte de la aceituna. El rabo tendría que ser siquiera para mí». Por eso te digo yo que aquella zona tiene lo que sea que le da los aromas a la aceituna (agricultor, Priego de Córdoba).

La falta de percepción de transformación a la producción de calidad por parte del sector contrasta con la implicación de todo el territorio, especialmente del sector turístico, en la difusión de la «cultura de la calidad». La DOP ha sido fundamental en la puesta en valor del territorio puesto que gran parte de su política va destinada a construir un sentimiento de identidad territorial en el que se involucren todos los sectores. "Priego es un caso curioso. De tal forma ha calado la DO, la calidad del aceite y su importancia como un elemento significativo de calidad de los Prieguenses. En pocos años se ha pasado a ser central. Pero ahora lo primero que te llevan es a ver la Fuente del Rey, y lo segundo que te hablan es de su historia y del aceite» (técnico, Priego de Córdoba).

En el modelo "Calidad como Expectativa de Futuro" existe una conciencia de estar en la fase más germinal e incipiente de un proceso de construcción de la calidad. Se alude a una labor lenta y difícil de concienciación de los agricultores y las industrias locales. Se hace referencia, incluso, a que serán las generaciones futuras las que se beneficiarán del esfuerzo actual. "Entonces es una lucha de concienciación a largo plazo. Exigir nos gusta a todos exigir mucho. Pero luego concienciarnos de eso va costando más trabajo» (representante institucional, Montoro-Adamuz).

Con todo, se advierten dos ejes sobre los que se articula el discurso sobre el modelo de calidad. Un eje gira en torno a la salud, ya que la variedad local de aceituna, el "nevadillo negro", es especialmente rica en polifenoles, uno de los importantes compuestos químicos que aporta las características saludables del Aceite de Oliva Virgen Extra. El segundo eje pone el acento en la tradición a la hora de destacar las cualidades diferenciales de su aceite. Se detectan grandes expectativas sobre un desarrollo territorial centrado en la puesta en valor del patrimonio asociado al aceite. De hecho, existen numerosos alojamientos rurales y restaurantes dispersos por la sierra, muchos de ellos antiguos molinos aceiteros rehabilitados.

Un rasgo diferencial de este territorio es la presencia de diferentes instituciones de conservación y promoción del olivar y el aceite: el «Museo del Aceite» de Montoro y el «Centro de Olivar de Sierra» de Adamuz. La presencia de estas entidades es una muestra del interés de este territorio por articular un modelo de producción de la calidad basado en la promoción del paisaje y el patrimonio olivarero y por convertir a estos elementos en su principal referente patrimonial e identitario y en el eje del desarrollo turístico del territorio. Sin que esta producción de calidad sea, por el momento, una realidad comarcal. 


\subsection{El discurso de la calidad del aceite de oliva. Valores asociados a la calidad desde el territorio}

Cada uno de estos territorios ha construido un relato específico para poner en valor el aceite de oliva. En este epígrafe, identificaremos cuáles son los elementos que aparecen en el discurso de los actores locales al hablar de la calidad de su aceite.

En el modelo "Calidad Corporativa», una empresa deviene en "voz territorial» que construye la memoria social hegemónica del territorio. Su historia, memoria y herencia simbólica son también los de la comarca, desvelando la importancia de valerse de un relato para cohesionar a la comunidad en torno al proyecto de calidad. La entidad construye una historia, con un pasado, presente y proyección de futuro. Con unos líderes fundadores, que se han convertido en personajes míticos de referencia, y con unos valores corporativos, ampliamente compartidos a nivel territorial.

A nivel de cultura material, no existe un patrimonio «visible» que dé cuenta del pasado olivarero reciente. Los antiguos molinos, o bien han desaparecido o constituyen un «patrimonio escondido" que está en manos privadas y que remite a un pasado de conflicto y desigualdad. Es decir, no se han socializado ni institucionalizado ni se han erigido como símbolos de la comunidad.

La piedra angular del relato de la calidad gira en torno a la esfera productiva del aceite, dejando a un lado la dimensión natural, paisajística, histórica, arqueológica, etc. Se pone en valor la extrema calidad de todo el aceite producido en la zona, su rigurosidad científico-técnica y la capacidad local de apertura al cambio cultural. El proceso de adaptación a la producción de calidad se vincula a la capacidad del territorio para apropiarse del conocimiento «experto» y para modernizar, tecnificar e intensificar la producción. También se enfatiza la tensión/diálogo entre la tradición representada por la cooperativa y la modernidad representada por la Cooperativa de Segundo Grado, así como el esfuerzo colectivo para superar las dificultades del proceso, lo que ha otorgado más fortaleza al proyecto. Algunos de los hitos conflictivos mencionados fueron: la dificultad para implantar el análisis sensorial, el sistema interno de pago por calidad, el envasado bajo una sola marca comarcal y, sobre todo, la cesión de autonomía cooperativa a favor del proyecto colectivo de la entidad líder.

En este modelo, el relato territorial se sustenta sobre el orgullo de haber sido capaces de generar una organización colectiva como estrategia de resistencia ante los procesos globalizadores de la cadena agroalimen- 
taria y como herramienta para luchar contra el fraude en la categorización de los aceites. Este relato de resistencia se extiende también a la creación de la entidad líder, ya que se narra que fue creada como estrategia colectiva ante la competencia insana interna propiciada por los corredores de aceite. Así mismo, la estrategia de calidad se percibe como una alternativa para trascender las desigualdades en la cadena del valor del aceite.

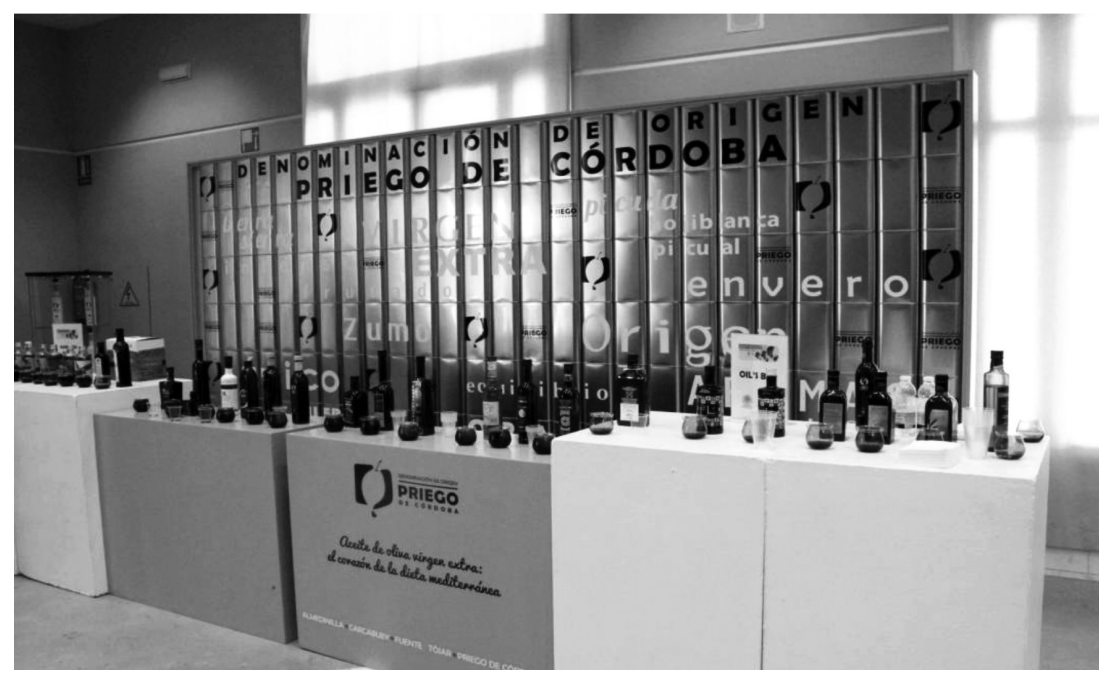

Foto 4. Muestra de la diversidad de marcas de aceites pertenecientes a la DOP Priego de Córdoba (elaboración propia).

En el modelo "Calidad Territorial» también es la entidad líder la que se erige como "voz territorial». Pero, en este caso, la que construye una memoria social es una entidad de derecho público que representa a una diversidad de iniciativas. El relato comarcal responde a dicha diversidad y pone en valor todas las dimensiones del territorio. Muestra de ello es que no existe un único mito de origen del proceso de conversión a la calidad ni un actor principal. Se reconoce, sin embargo, el protagonismo de la DOP, ya que esta refuerza tanto el liderazgo del sector privado como del cooperativo, así como la implicación del resto de instituciones locales.

Respecto a la cultura material, tampoco hay presencia de vestigios vinculados al pasado reciente que se erijan como símbolos. El patrimonio industrial está en manos privadas y responden también a un "patrimonio escondido" no institucionalizado. Ahora bien, se difunde la larga trayectoria del territorio en la producción de aceite de oliva a través de proyectos institucionalizados de patrimonialización que ponen en valor vestigios 
de época romana. Es importante señalar que, en la construcción de este discurso sobre la calidad, no solo han participado los integrantes del sector, sino también otros actores del territorio, entre los que destacan personas y asociaciones vinculadas a procesos de puesta en valor del patrimonio. El turismo también ha jugado un papel fundamental, porque el intercambio de impresiones con el visitante ha potenciado la puesta en valor del aceite como elemento patrimonial del territorio y el despliegue de narrativas sobre la identidad comarcal.

En este caso, el relato de la calidad se centra en las características edafológicas, climáticas y pluviométricas. Se alude a la altitud y la pendiente, e incluso a la flora específica del ecosistema del olivar de sierra. También se refiere a la variedad de aceituna mayoritaria, la picuda, así como al saber hacer de los industriales en la selección de la materia prima, la competencia sana entre las diferentes iniciativas, la capacidad de cooperación, intercambio e innovación colectiva entre ellas, la capacidad de cohesión de todo un territorio alrededor de un único proyecto de calidad territorial, el liderazgo compartido, la aportación colectiva a la imagen territorial, el renombre de la comarca adquirida con los premios, la apropiación local del conocimiento «experto» extendida a todo el territorio, así como el orgullo territorial por contar con reconocidos catadores de aceite.

Ahora bien, lejos de una visión romántica, se señala que, si los condicionantes orográficos no lo impidieran, el olivar tradicional se habría convertido a intensivo. Se percibe en los discursos un relato de doble resistencia: su menor productividad hace que este compita en desventaja tanto con el olivar intensivo enfocado al modelo de la cantidad, como al que se centra en el modelo de calidad «estandarizada».

En el modelo "Calidad como Expectativa de Futuro» no existe una entidad territorializada que lidere la conversión a la calidad, lo que explica la ausencia de una «voz territorial». Ahora bien, hay una memoria social fragmentada que concentran unos pocos actores, los que crearon la DOP Montoro-Adamuz, pero que no trasciende colectivamente al no existir una gobernanza territorial que difunda dicho relato.

En contraposición con los dos casos anteriores, destaca la importante presencia de cultura material vinculada al pasado reciente del «mundo del aceite». La población local es consciente de esta riqueza y menciona la gran cantidad de haciendas y cortijos que tuvieron o tienen una almazara en su interior, la presencia de un museo y de un centro del olivar, y la oferta turística local de casas rurales o restaurantes ubicados en haciendas rehabilitadas. Unas iniciativas de gran valor patrimonial, pero que están altamente infrautilizadas. 
Este modelo se caracteriza, como hemos mencionado, por un relato en el que la calidad se construye sobre las propiedades saludables de su variedad de aceituna, pero sobre todo en torno al valor del olivar tradicional de montaña, enfatizando su dimensión paisajística. Sin embargo, como en el segundo modelo, se hace referencia a la doble resistencia que debe afrontar este tipo de olivar respecto al intensivo. Se menciona la dificultad en la transformación de las prácticas agronómicas requeridas para producir calidad estandarizada en un olivar ubicado en extrema pendiente. En estas condiciones las fincas son difícilmente mecanizables y el desplazamiento diario a la almazara es complicado, lo que explica que en esta zona se mantengan prácticas agronómicas tradicionales.

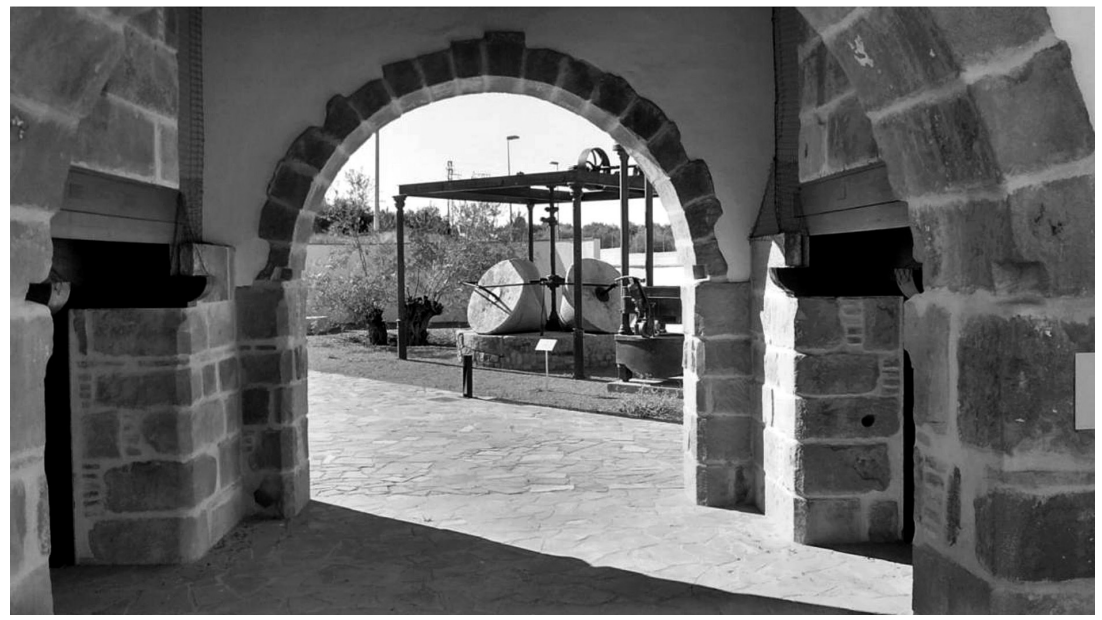

Foto 5. Instalaciones del «Centro del Olivar de Sierra» de Adamuz (elaboración propia).

\section{Conclusiones}

En este texto hemos analizado los cambios materiales y simbólicos que ha supuesto la implantación de un modelo de producción de calidad del aceite de oliva «reglamentada» basada en el conocimiento «científicotécnico". Hemos mostrado cómo estos discursos y prácticas son incorporados de manera diferencial por cada territorio en función de las especificidades de su contexto social, político, económico y cultural, dando lugar a distintos modelos, lo que nos ha permitido identificar tres itinerarios distintos en función de la entidad que ha liderado el proceso de construc- 
ción de la calidad, del papel que se ha otorgado al conocimiento «experto» en ese proceso y de las dimensiones a las que se ha otorgado preponderancia a la hora de construir el relato en torno a la calidad en cada uno de los territorios.

Los datos recabados en los tres casos de estudio muestran que, para que se produzca la conversión al modelo de calidad «reglamentada», es imprescindible la territorialización del conocimiento "experto". Resulta fundamental que el panel de cata pase a ser un actor activo del territorio y que ese conocimiento "experto" no esté restringido al conocimiento reglado de personas con titulaciones superiores. Al contrario, una parte importante del proceso se sustenta en la generación de un conocimiento accesible que se produce y difunde en y desde el territorio y que, a la vez, se nutre y retroalimenta con el conocimiento vernáculo del territorio. La capacidad de liderazgo, la pertenencia al territorio y el reconocimiento social que tengan las personas que impulsan el proyecto, así como el grado de identificación con la iniciativa y los lazos de confianza que se generen en torno al mismo son los elementos clave que hemos identificado.

De hecho, el elemento que los actores locales ponen en valor, y que es fuente de orgullo, es la singularidad del itinerario que cada territorio ha seguido en la transformación a la producción de calidad «estandarizada». Hemos visto que en el primer modelo se destaca la dimensión productiva del sector, en el segundo se potencia la puesta en valor de territorio y en el tercero toma especial relevancia la singular presencia de proyectos de patrimonialización institucionalizados. Un éxito que está vinculado al modo como se produce el diálogo entre lo local y lo global, entre la cultura local y las nuevas esferas de la cultura sociotécnica global, entre la tradición y la cultura empresarial y del mercado, entre el conocimiento tácito y el conocimiento codificado, entre un sentimiento de identidad territorial ya existente y el nuevo proceso de identificación que se pretende construir alrededor del proyecto.

\section{Referencias}

Aguilar, E. (2014). Los nuevos escenarios rurales: de la agricultura a la multifuncionalidad. Endoxa: Series Filosóficas, 33: 73-98.

Alimarket (2017). Informe aceite 2017. En https:/www.alimarket.es/alimentacion/infor me/229999/informe-2017-del-sector-de-aceites-en-espana. Accedido el 20 de enero de 2018.

Barea, F. y Ruiz Avilés, P. (2009). Estragegias de futuro para el sector oleícola andaluz. En Sostenibilidad de la producción de olivar en Andalucia. J.A. Gómez Calero, Coord. Sevilla. Junta de Andalucia. Consejería de Agricultura y Pesca. 
Belletti, G.; Marescotti, A. y Brazzini, A. (2014). Collective rules and the use of protected geographical indications by firms. International Agricultural Policy, 1: 11-20

Belletti, G.; Marescotti, A. y Touzard, J.M. (2017). Geographical Indications, Public Goods, and Sustainable Development: The Roles of Actors' Strategies and Public Policies. World Development, 97: 45-57.

Cendón, L. (2016). Efectos territoriales de las denominaciones de origen protegidas de aceite de oliva en España. Tesis doctoral no publicada, Escuela Técnica Superior de Ingenieros Agronomos, Universidad Politécnica de Madrid.

Cendón, L.; Sanz-Cañada, J. y Lucena-Piquero, D. (2014). Differential quality and technical/ managerial advice relationships in Andalusian (Spain ) olive oil protected designations of origin. Spanish Journal of Agricultural Research, 12(4): 869-888.

Colombo, S.; Villanueva Perujo, M. y Ruz Carmona, A. (2016). ¿Tienen futuro las pequeñas explotaciones olivareras tradicionales? Revista Olimerca Información de Mercados Para El Sector Del Aceite de Oliva y Otros Aceites Vegetales, 19: 34-39.

Consejería de Agricultura Pesca y Desarrollo Rural. (2015). Plan Director del Olivar Andaluz. Junta de Andalucia. En https://www.juntadeandalucia.es/export/drupaljda/ Plan\%20Director\%20del\%20Olivar.pdf. Accedido el 20 de enero de 2018.

Coq-Huelva, D.; Sanz-Cañada, J. y Sánchez-Escobar, F. (2014). Conventions, commodity chains and local food systems: Olive oil production in Sierra De Segura (Spain). Geoforum, 56: 6-16.

Fernández-Zarza, M.; Amaya-Corchuelo, S, y Aguilar-Criado, E. (en prensa). Institutional Density and Public Policies in two Cases of Geographical Indications from Mexico and Spain. Journal of Agrarian Change.

López-Moreno, I. y Aguilar, E. (2015). Resiliencia y cambios del sistema productivo en la agricultura familiar de la nueva ruralidad Europea: el caso del queso de oveja en la Sierra de Cádiz (España). Tessituras, Pelotos, 3(2): 242-268.

López Moreno, I.; Aguilar-Criado, E.; Lozano-Cabedo, C. y Pérez-Chueca, A. (2015). Quality labels and institutional density in the agro-food sector: the case of Andalusia (Spain). Spanish Journal of Rural Development, VI(3): 9-20.

Lozano Cabedo, C. (2013). Representaciones sociales de la agricultura ecológica en Andalucía. Gazeta de Antropología, 29(2). En http://www.gazeta-antropologia.es/?p=4306. Accedido el 30 de octubre de 2013.

Lozano Cabedo, C. (2011). El sabor de la naturaleza: agricultura ecológica en parques naturales andaluces. Sevilla: Fundación Blas Inflante.

Observatorio de precios y Mercados (2015). Informe final de la campaña 2014/2015 de aceite de oliva. Sevilla: Consejería de agricultura, pesca y desarrollo local.

Quiñones, X.F.; Penker, M.; Belleti, G.; Marescotti, A.; Scaramuzzi, S.; Barzini, E.; Pircher, M.; Leitgeb, F. y Samper-Gartner, L.F. (2016). Insights into the black box of collective efforts for the registration of Geographical Indications. Land Use Policy, 57: 103-116.

Rodríguez Cohard, J.C. y Parras-Rosa, M. (2012). Los canales de comercialización de los aceites de oliva españoles. Cuadernos de estudios agroalimentarios, 4: 93-102. 
Sánchez-Escobar, F. (2015). Sistemas complejos: una aplicación para el análisis de los balances energéticos y económicos en el agrosistema de olivar de Estepa. Tesis Doctoral no publicada. Departamento de Economía Aplicada II, Universidad de Sevilla.

Sánchez, J.; Garrido, A. y Paniza, A. (2017). Los olivares de montaña en la provincia de Jaén y sus desafios territoriales. AGER. Revista de Estudios Sobre Despoblación y Desarrollo Rural, 23: 1-36.

Sánchez Martínez, J. y Ortega Ruiz, A. (2016). El monocultivo olivarero jiennense: conformación histórica, valores patrimoniales y proyección cultural-turística. Cuadernos de Turismo, 37: 377-402.

Sanz-Cañada, J.; Hervás, I.; Sánchez-Escobar, F. y Coq-Huelva, D. (2014). Investigación e innovación en el sector del aceite de oliva en España. Problemas, oportunidades y prioridades de I+D+i. OLIVAE. Official Journal of the International Olive Council, 120: 31-47.

Sanz-Cañada, J. y Maciás Vaquez, A. (2005). Quality certification, institutions and innovation in local agro-food systems: Protected designations of origin of olive oil in Spain. Journal of Rural Studies, 21: 475-486. 